\title{
gु \\ Longitudinal bunch dynamics study with coherent synchrotron radiation
}

\author{
B. E. Billinghurst, ${ }^{*}$ J. C. Bergstrom, C. Baribeau, T. Batten, T. E. May, \\ J. M. Vogt, and W. A. Wurtz \\ Canadian Light Source Inc., University of Saskatchewan, Saskatoon, Saskatchewan S7N 2V3, Canada
} (Received 2 December 2015; published 23 February 2016)

\begin{abstract}
An electron bunch circulating in a storage ring constitutes a dynamical system with both longitudinal and transverse degrees of freedom. Through a self-interaction with the wakefields created by the bunch, certain of these degrees may get excited, defining a set of eigenmodes analogous to a spectroscopic series. The present study focuses on the longitudinal modes of a single bunch. The excitation of a mode appears as an amplitude modulation at the mode frequency of the coherent synchrotron radiation (CSR) emitted by the bunch. The modulations are superimposed on a much larger continuum from CSR emission in the continuous mode. A given eigenmode is classified by the integer $m$ which is the ratio of the mode frequency to the synchrotron frequency. The present measurements extend up to $m=8$ and focus on the region near the instability thresholds. At threshold the modes are excited sequentially, resembling a staircase when the mode frequencies are plotted as a function of bunch length or synchrotron frequency. Adjacent modes are observed to coexist at the boundaries between the modes. An energy-independent correlation is observed between the threshold current for an instability and the corresponding zero-current bunch length. Measurements were made at five beam energies between 1.0 and $2.9 \mathrm{GeV}$ at the Canadian Light Source. The CSR was measured in the time domain using an unbiased Schottky diode spanning 50-75 GHz.
\end{abstract}

DOI: 10.1103/PhysRevAccelBeams.19.020704

\section{INTRODUCTION}

Synchrotron radiation emitted by a relativistic electron bunch circulating in a storage ring is usually incoherent due to the random phases of the fields from each electron. However, when the bunch length, or a substructure within it, is sufficiently short relative to the wavelength of the radiation, the electron fields are nearly in phase with each other, resulting in coherent synchrotron radiation (CSR). Since the coherent fields add linearly with a common phase, the spectral intensity can be extremely large relative to the corresponding incoherent synchrotron radiation.

The temporal nature of the CSR is a strong function of the bunch charge. For a sufficiently low charge, the longitudinal charge density assumes a static Gaussian-like profile as defined by the Haissinski equation [1]. The spectral intensity of the CSR as a function of wavelength is determined by the Fourier spectrum of the charge density over the same wavelength region. Since this distribution is constant on a turn-by-turn basis, the CSR is said to be emitted in the continuous mode.

With increasing charge, the internal dynamics of the bunch becomes a complex function of time. A microwave instability generates rapidly evolving microstructures

\footnotetext{
*brant.billinghurst@lightsource.ca

Published by the American Physical Society under the terms of the Creative Commons Attribution 3.0 License. Further distribution of this work must maintain attribution to the author(s) and the published article's title, journal citation, and DOI.
}

within the charge distribution that become sources of CSR. These microstructures, or microbunches, evolve from a reaction between the electrons and their own CSR wakefields, producing a runaway positive feedback mechanism [2,3]. Higher order damping processes eventually limit the explosive growth of these microbunches and return the system to its starting point, where the whole process begins anew. The time interval between these CSR bursts is irregular and therefore the spectral intensity varies on a turn by turn basis. This is referred to as CSR in the bursting mode.

A transition region exists between the continuous and bursting modes where the CSR intensity oscillates harmonically in time at frequencies typically in the range $10-100 \mathrm{kHz}$. The periodic nature of the CSR in this region is driven by the longitudinal normal modes of oscillation within the electron bunch. The corresponding eigenmode frequencies are harmonics of the synchrotron frequency, and are the focus of the present study. A historic summary of the observation of bunch instabilities through a study of CSR is given in [4].

It is useful to picture the longitudinal dynamics in the transition region within the framework of a Cartesian phase space $(q, p)$, where the vertical coordinate $p$ is proportional to the energy deviation $E-E_{0}$ of a given electron from its mean value and the horizontal coordinate $q$ is its longitudinal position within the bunch. With appropriate normalization of the $p$-axis, the phase-space motion of an electron below the instability threshold and well within the rf bucket is a closed circular path rotating at the synchrotron 
frequency $F_{\text {syn }}$ as defined by the parameters of the rf cavity and the storage ring. The entire quiescent bunch maps into a fixed circular pattern in phase space, rotating at $F_{\text {syn }}$. The density of particles within this phase space is best expressed in circular coordinates $(\tau, \varphi)$ where the azimuthal angle $\varphi$ is measured from the $q$-axis and the "radius" $\tau$ is the synchrotron amplitude of a given particle. The Cartesian and circular representations are related by $q=\tau \cos (\varphi)$ and $p=\tau \sin (\varphi)$. In general, we can write the phase-space density as a Fourier expansion in $\varphi[5]$ :

$$
\rho(\tau, \varphi)=e^{-i \omega_{s} t} \sum_{m=-\infty}^{\infty} \alpha_{m} R_{m}(\tau) e^{i m \varphi}
$$

which rotates at the synchrotron frequency $\omega_{s}=2 \pi F_{\text {syn }}$. The integers $m$ define the periodicity of $\rho(\tau, \varphi)$ with respect to $\varphi$, and are termed azimuthal modes since they correspond to phase-space distortions in the $\varphi$-direction. The constants $\alpha_{m}$ define the relative contribution of a given mode to the density, and for a quiescent bunch only the $m=0$ term survives. For a given azimuthal mode $m$, the functions $R_{m}(\tau)$ can be further expanded around a set of functions describing eigenmodes along the radial coordinate $\tau$ : $R_{m}(\tau)=\sum_{n} \beta_{m, n} \rho_{m, n}(\tau)$. The radial phase-space distortions $\rho_{m, n}(\tau)$ are termed radial modes, and are degenerate in energy in the absence of wakefield interactions. The constants $\beta_{m, n}$ define the contribution of the radial mode $n$ to the density for a given azimuthal mode $m$. Each azimuthal mode, characterized by its unique excitation energy, can be accompanied by one or more degenerate radial modes.

As we cross the instability threshold, the lowest excited azimuthal mode is the quadrupole mode defined by $m=2$ (we exclude the $m=1$ dipole mode corresponding to a synchrotron oscillation of the entire bunch). The $m=2$ phase-space distortion defined by Eq. (1) is proportional to $\cos (2 \varphi)$ in a simple qualitative picture. This distortion rotates at the synchrotron frequency so the total pattern appears to repeat itself at a frequency $2 F_{\text {syn. The next }}$ excitation is the $m=3$ sextupole mode, described by $\cos (3 \varphi)$, and the phase space pattern replicates at a frequency of $3 F_{\text {syn }}$ (we are ignoring here the possibility that different modes might coexist). In general, an excited azimuthal mode of order $m$ can be crudely visualized in phase space as a star-shaped configuration with $m$ prongs described by $\cos (m \varphi)$, rotating at the synchrotron frequency so the associated mode frequency becomes $m F_{\text {syn }}$. By way of example, in Fig. 1 we show the phase space density for modes $m=3$ and $m=8$ in the cosine description with a Gaussian radial dependence. The projection of the prongs onto the spatial $q$-axis defines the periodic density fluctuations within the bunch, analogous to the microbunches described above [6,7]. The quadrupole mode, for example, appears as a longitudinal "breathing" mode of the bunch where the bunch length appears to oscillate at $2 F_{\text {syn }}$ and the CSR intensity oscillates accordingly.
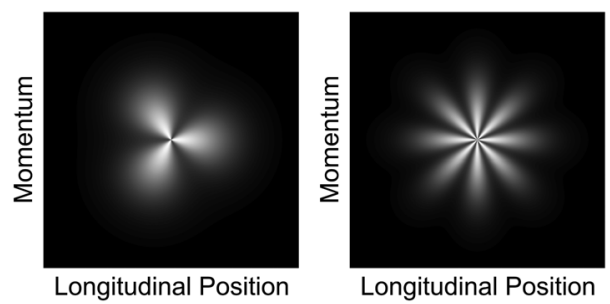

FIG. 1. Models of the phase space densities for modes $m=3$ (left) and $m=8$ (right) assuming a $\cos (m \varphi)$ azimuthal distortion with a Gaussian radial dependence. The patterns rotate at the synchrotron frequency $F_{\text {syn }}$ and the mode frequencies are $m F_{\text {syn }}$.

The interaction of the bunch with its own wakefields can induce a shift in the synchrotron frequency and thus cause a shift in the frequencies of the azimuthal modes relative to the above simplistic model. Two phenomena dominate, and tend to act in opposite directions. Potential-well distortion [8], a static mechanism, causes an increase in the bunch length and a decrease in the synchrotron frequency. Consequently, the mode frequencies are shifted downwards. The other mechanism is dynamic. In the linear theory the deviation from the static Haissinski profile is assumed to vary as $\rho(z) e^{-i \Omega_{m} t}$ where $\Omega_{m}=2 \pi m F_{\text {syn }}$ and $\rho(z)$ represents the longitudinal deviation. The wakefield produced by this deviation constitutes a time-varying retarding voltage acting on the bunch. The net result is an upward shift in the synchrotron frequency in the presence of an inductive impedance [5], and thus an upward shift in the mode frequency. Both of these phenomena are current-dependent. The $m=1$ dipole mode, corresponding to the familiar synchrotron oscillation of the entire bunch, is the exception here. For this mode, the two mechanisms completely cancel each other, leaving this frequency unchanged $[5,8]$. In the following, we will refer to this as the "true" synchrotron frequency.

We turn now to the instability thresholds, and consider the bunch current as the independent parameter. Threshold calculations generally follow two approaches. For example, Bane et al. [9] solve the Vlasov-Fokker-Planck equation and note the onset of instability through a change in the rms energy spread of the bunch. This leads to a convenient threshold algorithm which we will consider later. The other approach is based on a linearization of the Vlasov equation and uses the concept of mode coupling to describe the instability. With increasing current, certain mode frequencies converge (azimuthal or radial) and the mode frequency acquires an imaginary component which describes the instability growth rate. The recent work of Cai [10] exemplifies this approach.

Let us now briefly review previous experimental CSR studies in the transition region in the time domain. We use the symbol $F_{\text {inst }}$ to represent the instability frequency as defined by the harmonic variation of the CSR intensity, and $F_{\text {syn }}$ to represent the true synchrotron frequency. If $F_{\text {inst }}$ 
corresponds to the excitation of the azimuthal modes, a plot of $F_{\text {inst }} / F_{\text {syn }}$ should exhibit a clustering around the integral values appropriate to the excited modes. This is indeed nicely demonstrated in the work of Kuske [11], which shows a distinct clustering, staircase-fashion, about the three lowest modes $(m=2-4)$ when $F_{\text {inst }} / F_{\text {syn }}$ is plotted as a function of $F_{\text {syn }}$. With increasing mode number, the stairlike platforms develop an upward tilt and the discontinuities are less evident although the measurements extend up to $m \approx 7$. These pioneering measurements were made at $1.7 \mathrm{GeV}$ on the BESSY II synchrotron. Threshold studies have subsequently been reported by others [12-14], but they do not focus on excitations above the sextupole mode $(m=3)$.

In this paper we present our experimental investigations of longitudinal bunch dynamics in the transition region through a study of CSR in the time domain using the Canadian Light Source storage ring. Data were acquired with a Schottky diode in the $\mathrm{mm}$ region of the CSR spectrum. Clear evidence of excitations up to the $m=8$ azimuthal mode is presented, with lesser evidence of excitations up to $m=11$. The instability threshold currents are compared with the theories of Bane et al. [9] and Cai [10]. We investigate these bunch characteristics as a function of five beam energies between 1.0 and $2.9 \mathrm{GeV}$.

\section{EXPERIMENTAL DETAILS}

CSR was produced at the Canadian Light Source (CLS) in Saskatoon in a single-bunch mode at five beam energies: $2.9,2.2,1.5,1.2$, and $1.0 \mathrm{GeV}$. Starting at a bunch current of 1-2 mA, the momentum compaction of the lattice was reduced until CSR emission was signaled by the huge increase in intensity associated with the transition from incoherent to coherent radiation. At a fixed synchrotron frequency, the bunch current was then slowly reduced by beam scrapers until the CSR transitioned from the stochastic bursting mode to a steady periodic mode. The nominal threshold current for periodic emission was subjectively defined when the oscillation amplitude was small relative to the underlying continuous spectrum but still clearly visible, which was about $5 \%$ or more of the continuum amplitude. The momentum compaction and synchrotron frequency were again reduced and the cycle repeated until limited by the short beam lifetime at small compaction factors. The true synchrotron frequency $F_{\text {syn }}$ near threshold was deduced in the usual manner from the $m=1$ synchrotron sideband on a storage-ring rotation harmonic as measured by a beam position monitor.

The CSR was detected by an unbiased rf Schottky diode equipped with a $50-75 \mathrm{GHz}$ band pass filter [15]. The diode was mounted in air at the end of a $50 \mathrm{~cm}$ long forward-viewing pipe fastened near the entrance of the bending-magnet vacuum chamber $(R=7.143 \mathrm{~m}$, bend angle $\theta=15^{\circ}$, height $H=32 \mathrm{~mm}$ ). The detector thus observes backward-propagating fields generated by

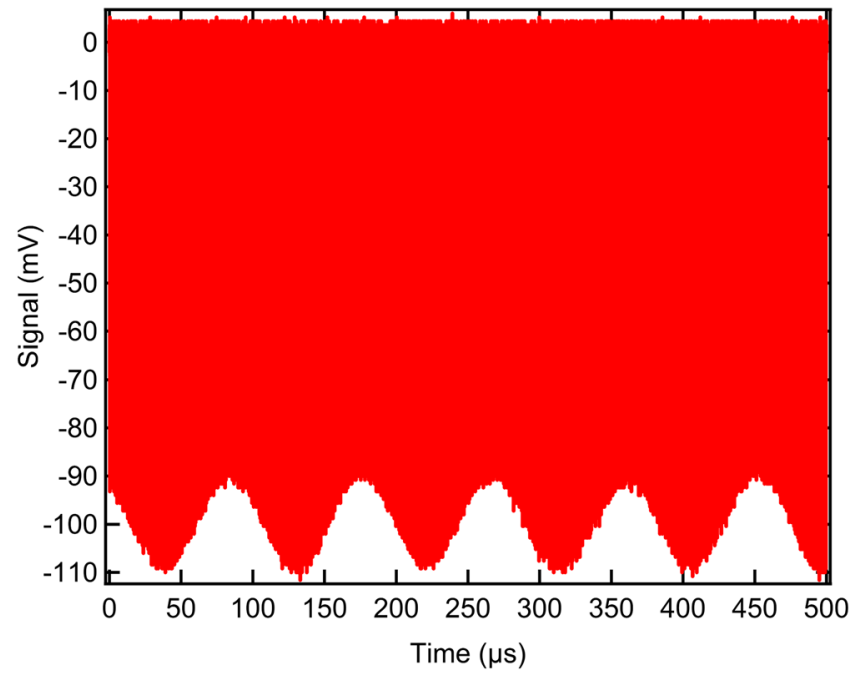

FIG. 2. Time-domain CSR spectrum showing oscillations due to the quadrupole $(m=2)$ eigenmode near threshold. The oscillation frequency is approximately $10.9 \mathrm{kHz}$ and the synchrotron frequency is $5.4 \mathrm{kHz}$. The figure represents 877 turns of the storage ring.

reflections from a photon-absorber bar and other components situated near the vacuum chamber exit. The layout is described in more detail in Ref. [16].

A typical time-domain spectrum near the periodic threshold is shown in Fig. 2, spanning $500 \mu$ s, or 877 passes around the storage ring. The modulation frequency here is $10.9 \mathrm{kHz}$, and is associated with the quadrupole $(m=2)$ longitudinal mode. Figure 3 shows the detector response for a single pass of the bunch. The light from each pass is comprised of the prompt CSR flash plus contributions from the trailing wakefields and reflections from other hardware components, as described in [16].

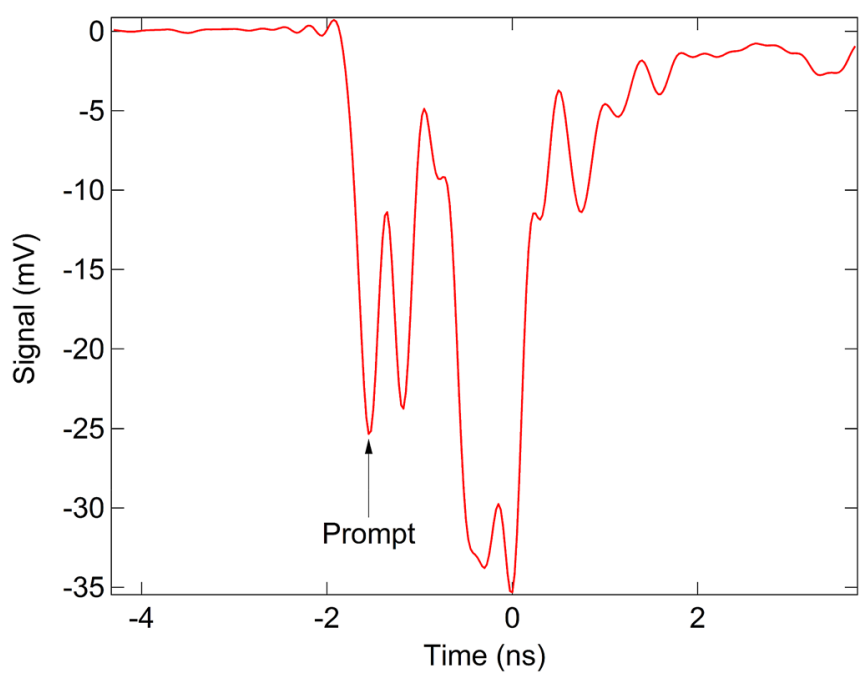

FIG. 3. Detector response for a single pass of the bunch. In this study we are only interested in the prompt peak. The meaning of the other structures is described in [16]. 
Data were analyzed using a custom procedure written in Igor 6.3.4.0. For this study we are interested only in the prompt peak. The analysis software picked the maximum intensity of this peak for each pass. The DC component was removed from the resulting multipass set of amplitudes and a Fast Fourier Transform (FFT) was performed. The FFT was zero filled [17] to 16384 points and used a Blackman window function [18]. Peaks in the resulting frequency spectrum were selected based on their intensity maxima.

\section{RESULTS AND DISCUSSION}

\section{A. Instability frequency as a function of bunch length}

The independent experimental parameters in this study are the bunch current $I_{b}^{\mathrm{thr}}$ defining an eigenmode threshold, the true synchrotron frequency $F_{\text {syn }}$, and the beam energy. Note that $I_{b}^{\text {thr }}$ is the eigenmode threshold current for a given synchrotron frequency, since these two parameters are correlated. All the data reported herein correspond to threshold measurements spanning this 2-dimensional space. We will mainly confine our discussion to two beam energies, 1.5 and $2.9 \mathrm{GeV}$. These two energies display a marked difference in the profile of their respective excitation functions, and we will attempt to address the origin of this difference. We will comment on the other energies when appropriate.

In Fig. 4 we plot the excitation function $F_{\text {inst }} / F_{\text {syn }}$ as a function of the threshold current $I_{b}^{\mathrm{thr}}$, where $F_{\text {inst }}$ is the

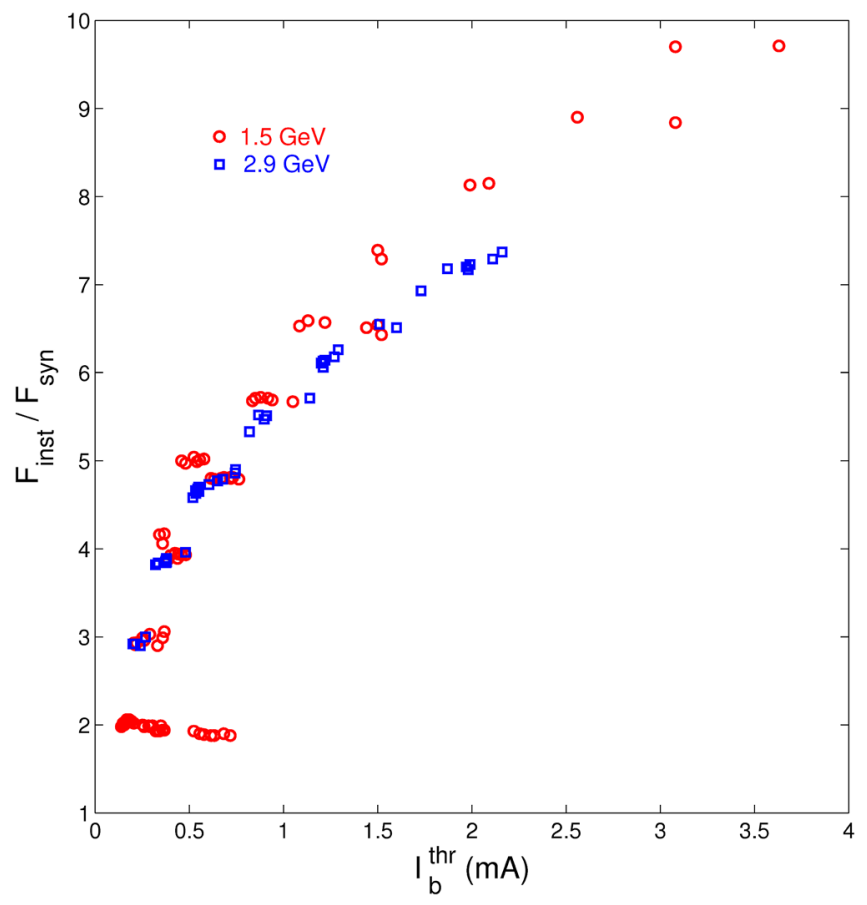

FIG. 4. Instability frequency normalized by the true synchrotron frequency as a function of the threshold bunch current at 1.5 and $2.9 \mathrm{GeV}$. A tight clustering around the lower integers is apparent. instability frequency as determined above. A tight clustering around integer values is observed, while a downward drift below integral values is seen with increasing current. It is also apparent that $F_{\text {inst }} / F_{\text {syn }}$ is not a simple linear function of the threshold current. On the other hand, elementary considerations suggest that $F_{\text {inst }} / F_{\text {syn }}$ should be a linear function of the bunch length, with a slope that is independent of energy [7]. Let us briefly review the argument. With reference to the phase-space model in our introductory remarks, the angle between the prongs of the phase-space "star" is given by $\Delta \varphi=2 \pi F_{\text {syn }} / F_{\text {inst }}$. Since the radius of the star is approximately equal to the bunch length $\sigma$, the projection of the star onto the spatial $q$-axis defines the maximum spacing $\lambda_{0}$ between the resulting density maxima as $\lambda_{0} \approx \sigma \Delta \varphi$. Combining these expressions we get

$$
\frac{F_{\text {inst }}}{F_{\text {syn }}} \approx \frac{2 \pi \sigma}{\lambda_{0}}
$$

A density modulation of wavelength $\lambda_{0}$ translates into CSR of the same wavelength, so we identify $\lambda_{0}$ with the CSR spectral maximum in the $\mathrm{THz}$ region. Kuske [19] came to a similar conclusion as Eq. (2), and suggested that $\lambda_{0}$ be defined as the wavelength corresponding to the local maximum in the longitudinal radiation impedance $\operatorname{Re}[Z(\omega)]$, using a broad-band resonance approximation to the parallel-plate impedance as derived by Warnock [20]. This gives

$$
\lambda_{0} \approx\left[\frac{3 H^{3}}{\pi R}\right]^{\frac{1}{2}}
$$

where $H$ is the height of the chamber and $R$ is the bending radius. For the CLS parameters, we get $\lambda_{0}=2.09 \mathrm{~mm}$. Finally, combining (2) and (3) gives the required expression. We calculate the zero-current bunch length $\sigma$ using the relation

$$
\sigma=\frac{c F_{\text {syn }}}{F_{0}^{2}} \cdot \frac{E}{h V_{0} \cos (\psi)} \cdot \frac{\Delta E}{E}
$$

where $F_{0}$ is the rotation frequency $(1.754 \mathrm{MHz}), c$ is the speed of light, $h$ is the harmonic number (285), $V_{0}$ is the cavity voltage $(2.05 \mathrm{MV}), \psi$ is the stable phase, and $\Delta E / E$ is the relative energy spread of the beam as calculated from the synchrotron radiation integrals.

In Fig. 5 we show $F_{\text {inst }} / F_{\text {syn }}$ plotted as a function of the zero-current bunch length for 1.5 and $2.9 \mathrm{GeV}$. The straight line in the figure is based on Eqs. (2) and (3). The trend of the data is indeed linear with $\sigma$, and the general slope is consistent with $2 \pi / \lambda_{0}$ as given by Eq. (2), independent of energy. Of particular note in Fig. 5 is the apparent alignment of the line with the start of each mode. This empirical observation that seemingly ties a given modal threshold to a 


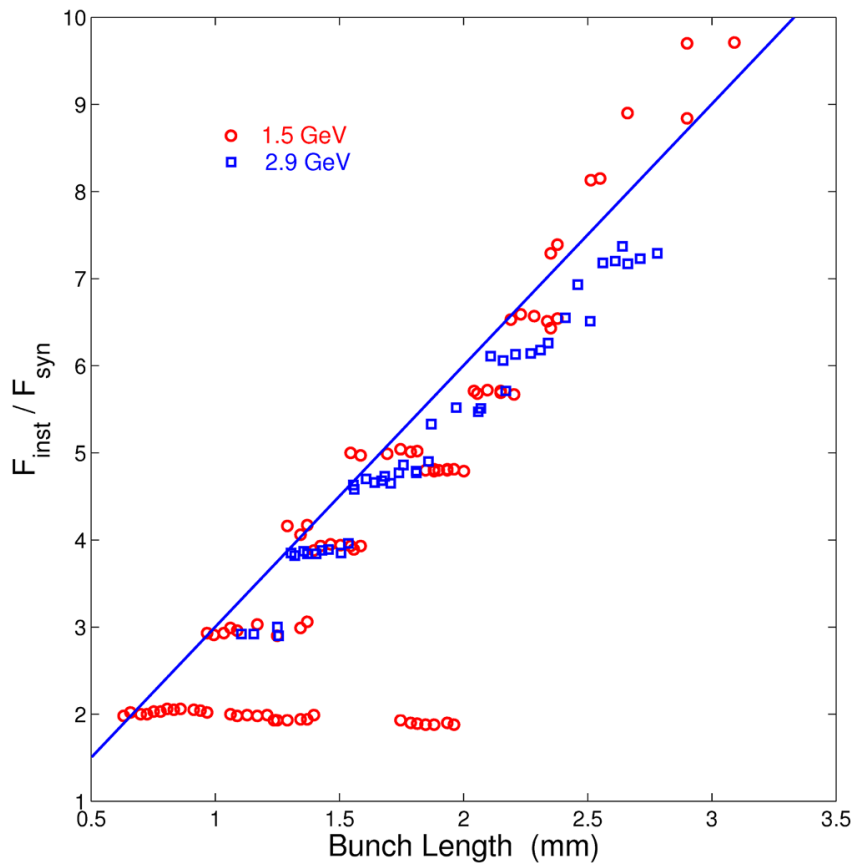

FIG. 5. Instability frequency normalized by the true synchrotron frequency as a function of the zero-current bunch length at the same synchrotron frequency. Beam energies are 1.5 and $2.9 \mathrm{GeV}$. A linear dependence on the bunch length is evident, supporting the elementary argument in the text. The straight line is the corresponding prediction based on Eqs. (2) and (3).

unique bunch length will be addressed later in the threshold discussion.

\section{B. Instability frequency as a function of synchrotron frequency-1.5 GeV}

In Fig. 6 we show $F_{\text {inst }} / F_{\text {syn }}$ at threshold as a function of the synchrotron frequency for beam energies $2.9,2.2$, and $1.5 \mathrm{GeV}$, while Fig. 7 shows the corresponding plots at 1.2 and $1.0 \mathrm{GeV}$. All the frequency ratios fall close to, or below, the integer mode numbers $m$ and the downward shifts are seen to increase with increasing $m$, a trend that is seen to continue at the lower beam energies as well. We also note that the 2.9 and $1.5 \mathrm{GeV}$ excitation functions differ in their profiles across each mode. The $1.5 \mathrm{GeV}$ (and lower energy) data form a staircase-like pattern with horizontal steps for each mode, while the $2.9 \mathrm{GeV}$ data show a distinct upward slope across each step. The $2.2 \mathrm{GeV}$ data hint at a transition between the two. Let us comment first on the $1.5 \mathrm{GeV}$ measurements in Fig. 6.

Bunch instabilities are often described in terms of mode coupling between the radial modes or the azimuthal modes [8]. The resulting eigenmode frequencies change with increasing current, and if they eventually overlap then the resulting frequency acquires an imaginary component that defines the instability threshold and the growth rate. In general, the growth rate is much smaller for radial-mode coupling than azimuthal-mode coupling, but both predict a

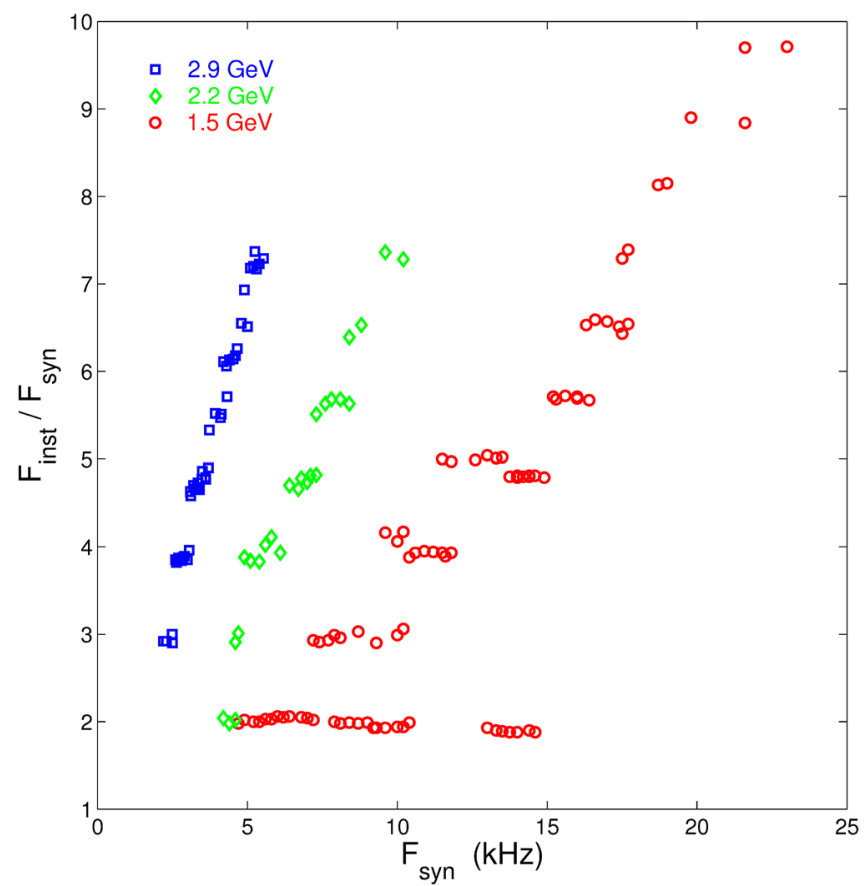

FIG. 6. Instability frequency normalized by the true synchrotron frequency as a function of the true synchrotron frequency for beam energies 2.9, 2.2 and $1.5 \mathrm{GeV}$. Excitation of eigenmodes up to $m=11$ is evident in the $1.5 \mathrm{GeV}$ data.

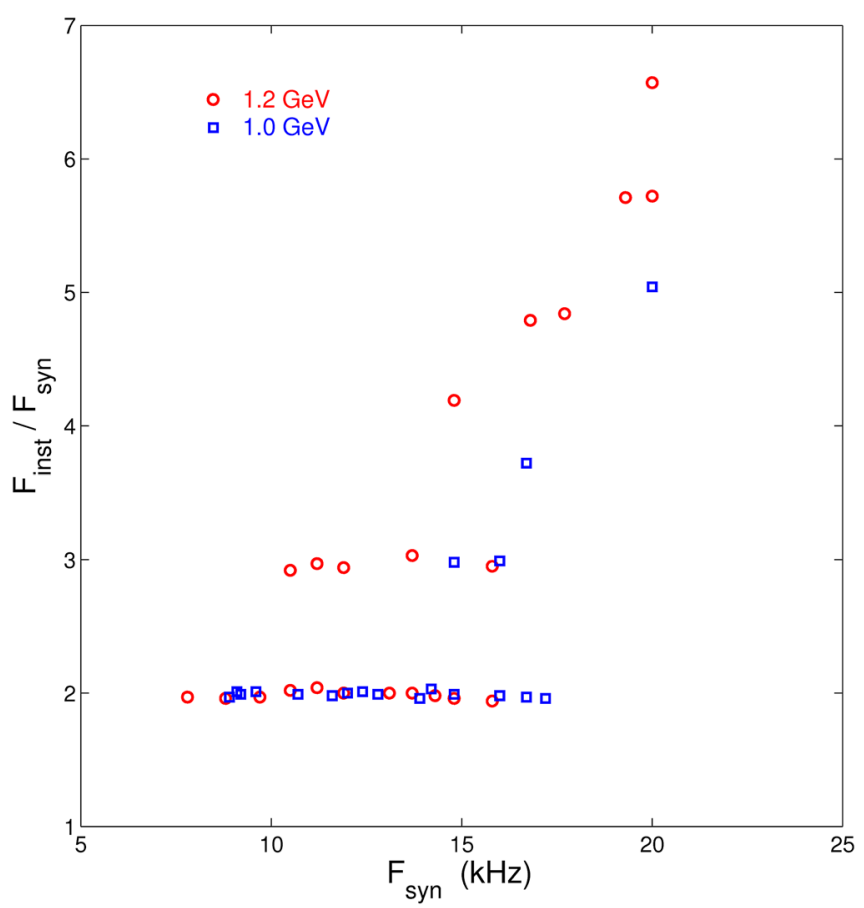

FIG. 7. Instability frequency normalized by the true synchrotron frequency as a function of the true synchrotron frequency for beam energies 1.2 and $1.0 \mathrm{GeV}$. 
very rapid increase in growth rate with increasing current above threshold.

The frequencies of the unperturbed radial modes are identical to those of the associated azimuthal modes, so radial-mode coupling for a given azimuthal mode produces a relatively small shift in the eigenmode frequencies. In contrast, coupling between azimuthal modes (for a fixed radial mode) produces a large shift, comparable to $F_{\text {syn }}$ [8,10,21]. Inspection of the $1.5 \mathrm{GeV}$ data in Fig. 6 shows negligible or small shifts from integral values up to $m=5$, suggesting that coupling between radial modes plays the dominant role here. This conjecture is supported by detailed calculations, with as few as two radial modes participating [10].

The normalized frequencies in Fig. 6 gradually slip below integral values with increasing $m$. We associate these downward shifts with a reduction in the synchrotron frequency caused by potential-well distortion (PWD). Denoting this frequency shift by $\Delta F_{\text {pwd }}$, the frequency of a given mode becomes $F_{\text {inst }}=m\left(F_{\text {syn }}+\Delta F_{\text {pwd }}\right)$ where $F_{\text {syn }}$ is the true synchrotron frequency. The PWD-induced frequency shift is a negative quantity in the presence of an inductive impedance [8], therefore $F_{\text {inst }} / F_{\text {syn }}$ should shift below integer values with increasing $m$, in agreement with the general trend of the $1.5 \mathrm{GeV}$ data.

We have made a rough estimate of the PWD correction to the synchrotron frequency using the approximation [8]:

$$
\Delta \omega_{s} \approx \frac{\alpha c^{2} r_{0}}{4 \pi \omega_{s} \gamma C} \int \rho(\omega) \frac{\omega}{c} \operatorname{Im}[Z(\omega)] d \omega .
$$

Here, $\alpha$ is the momentum compaction, $r_{0}$ is the classical electron radius, $\gamma=E / m_{e}, C$ is the storage ring circumference, and $\Delta \omega_{s}=2 \pi \Delta F_{\mathrm{pwd}}$. The bunch density is approximated by a Gaussian: $\rho(\omega)=N_{b} e^{-(\omega \sigma / c)^{2} / 2}$, and the bunch length $\sigma$ is corrected for PWD using the relation $\left(\sigma \omega_{s}\right)=\left(\sigma \omega_{s}\right)_{0}$ where the right side means unmodified quantities [22]. The impedance $Z(\omega)$ is based on the parallel-plate model [20] evaluated for the CLS geometry. Finally, we express the momentum compaction in Eq. (5) in terms of the cavity voltage, since the voltage and phase are maintained at fixed values for all beam energies:

$$
\alpha=2 \pi E \cdot\left[\frac{F_{\mathrm{syn}}}{F_{0}}\right]^{2} \cdot \frac{1}{h V_{0} \cos (\psi)} .
$$

Combining Eqs. (5) and (6), we note that the expression for $\Delta F_{\text {pwd }} / F_{\text {syn }}$ now becomes independent of both energy and synchrotron frequency.

The PWD shift was calculated for the modes $m=5-7$ using the bunch parameters associated with the corresponding $1.5 \mathrm{GeV}$ measurements. In all cases we estimate $\Delta F_{\text {pwd }} / F_{\text {syn }} \approx-0.08$ while the experimental shifts as deduced from the data are $\left(\Delta F_{\text {inst }} / F_{\text {syn }}\right)_{\exp } \approx-0.035$, -0.048 and -0.060 for $m=5-7$, respectively. The variation in the experimental shifts can be ascribed to the corresponding differences in the upward shifts associated with the so-called dynamic correction to the synchrotron frequency described in the introductory remarks. The instability frequency corrected for both PWD and the dynamic term becomes $F_{\text {inst }}=m\left(F_{\text {syn }}+\Delta F_{\text {pwd }}+\Delta F_{\text {dyn }}\right)$. If we adopt the PWD estimate, it follows that the dynamic shifts must decrease rapidly in magnitude with increasing mode number in order to reproduce the experimental shifts, in accord with theoretical expectations $[5,8]$. Consequently, the total synchrotron frequency shift $\Delta F_{\text {pwd }}+\Delta F_{\text {dyn }}$ must decrease in magnitude with decreasing mode due to a growing cancellation between the two terms, with complete cancellation occurring for the dipole $(m=1)$ mode. This competing behavior between the static and dynamic corrections accounts for the pattern of frequency shifts displayed in Fig. 6 as a function of mode number.

\section{Instability frequency as a function of synchrotron frequency-2.9 GeV}

We turn now to the $2.9 \mathrm{GeV}$ measurements and focus on the upward slopes across each mode. These data are displayed in an expanded scale in Fig. 8, where the lines through the data are merely a visual guide. Interestingly, the frequency ratio $F_{\text {inst }} / F_{\text {syn }}$ at the high-frequency end of each mode closely matches the corresponding $1.5 \mathrm{GeV}$ frequency ratio for the same mode number (see Fig. 6), suggesting comparable PWD and dynamic corrections for

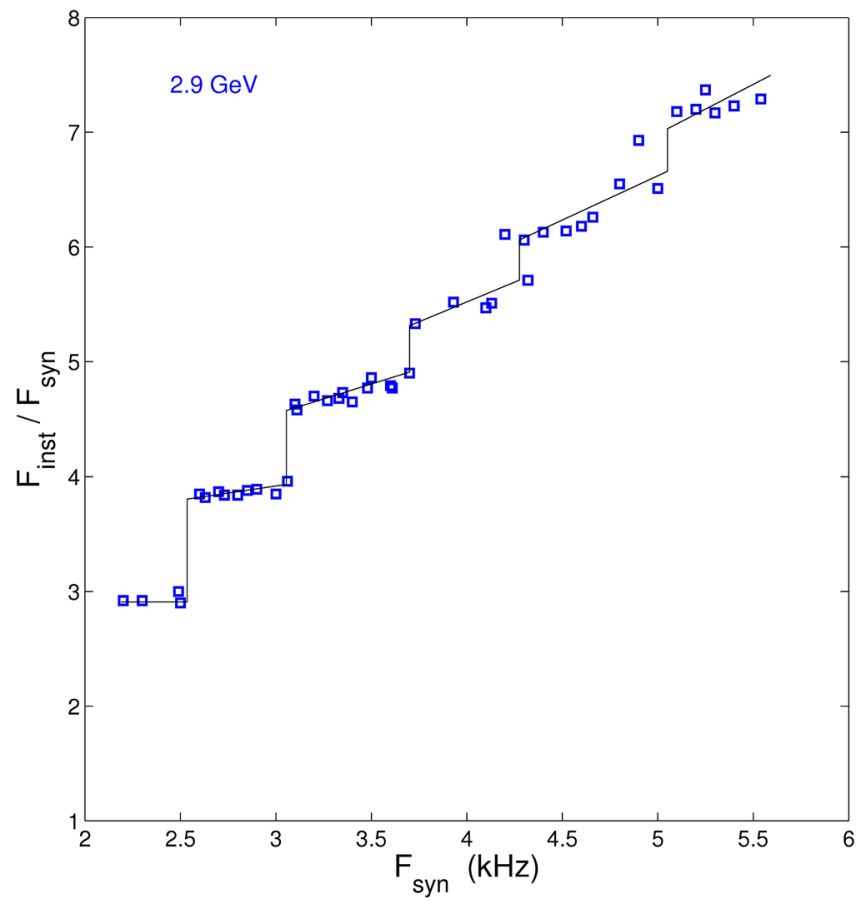

FIG. 8. The $2.9 \mathrm{GeV}$ data of Fig. 6 in expanded scale showing an upward slope across each mode that increases with increasing mode number, in marked contrast to the behavior at $1.5 \mathrm{GeV}$. The lines through the data are merely a visual guide. 
the two sets of measurements. This is plausible since we have argued that the normalized PWD shift $\Delta F_{\text {pwd }} / F_{\text {syn }}$ is independent of both energy and synchrotron frequency for our operating mode, and one can make a similar argument for the normalized dynamic correction $\Delta F_{\mathrm{dyn}} / F_{\mathrm{syn}}$. On the other hand, at the low-frequency end of each $2.9 \mathrm{GeV}$ mode, we see that $F_{\text {inst }} / F_{\text {syn }}$ falls well below the $1.5 \mathrm{GeV}$ results (see Fig. 6). Figure 6 clearly suggests some kind of correlation between the slopes and $F_{\text {syn }}$, given the large difference in frequencies between 1.5 and $2.9 \mathrm{GeV}$. As further evidence, we note that the BESSY II data [11], which also exhibits upward slopes across each mode, was accumulated at $1.7 \mathrm{GeV}$, comparable our $1.5 \mathrm{GeV}$ energy. However, the corresponding synchrotron frequencies of approximately $1-8 \mathrm{kHz}$ are much closer to the frequencies 2-6 kHz we measured at $2.9 \mathrm{GeV}$ than to the much higher frequencies measured at $1.5 \mathrm{GeV}$. In other words, the BESSY II measurements of $F_{\text {inst }} / F_{\text {syn }}$ vs. $F_{\text {syn }}$ bear a much closer resemblance to our $2.9 \mathrm{GeV}$ results than to our $1.5 \mathrm{GeV}$ results, again suggesting a correlation between the slopes and $F_{\text {syn }}$.

We suggest that the apparent correlation between the upward slopes and $F_{\text {syn }}$ may be related to radiation damping within the bunch. The synchrotron period $\tau_{\mathrm{syn}}=$ $1 / F_{\text {syn }}$ defines the natural time unit for the internal bunch dynamics. Denoting the longitudinal damping time by $\tau_{d}$, the ratio $B=\tau_{\text {syn }} / \tau_{d}$ provides a relative measure of the damping effect on the bunch dynamics. From the known damping times and the central frequencies in Fig. 6, we estimate $B \approx 0.10$ at $2.9 \mathrm{GeV}$ and $B \approx 0.004$ at $1.5 \mathrm{GeV}$ which differ by a factor of 25 , suggesting that damping is probably not a factor at the lower energy. For the BESSY II measurements we estimate $B \approx 0.04$ which is much closer to our $2.9 \mathrm{GeV}$ value than our $1.5 \mathrm{GeV}$ value. Thus, $B$ provides a mechanism for the observed correlation with $F_{\text {syn }}$. Damping is important when the instability growth time is comparable to $\tau_{d}$, and given that the growth times are generally many synchrotron periods, the damping influence should increase with $B$ and therefore should be most apparent at $2.9 \mathrm{GeV}$. Each eigenmode "step" in Fig. 6 and Fig. 8 is defined by a threshold current or threshold synchrotron frequency that marks the initiation of the mode. Assuming that the instability growth rates increase rapidly above these thresholds, damping should have a maximum impact near threshold and its influence should decrease across the step of a given mode. The dynamic correction $\Delta F_{\text {dyn }}$ by its nature will be more sensitive than the static PWD to radiative damping in the threshold region. This balance between damping and excitation means $\Delta F_{\mathrm{dyn}}$, which is a positive quantity, will be smaller near the eigenmode threshold than at the higher currents where the next higher mode begins, thus contributing to an upward slope in $F_{\text {inst }} / F_{\text {syn }}$. Finally, we note that $B / \pi$ appears as a coefficient multiplying the damping term in the Vlasov-Fokker-Planck (VFP) equation. Kuske [23] has calculated $F_{\text {inst }} / F_{\text {syn }}$ vs. $F_{\text {syn }}$ for the BESSY II parameters using the full VFP equation and predicts upward slopes across the steps similar to the BESSY II measurements (see also [19]).

We can put the preceding remarks in more quantitative terms by considering the synchrotron frequency shifts for a parabolic charge distribution, which is much more tractable than the Gaussian model while still demonstrating the essential features. For simplicity, the impedance is taken to be purely inductive $(Z(\omega)=-i \omega L)$ and only the most prominent radial mode is included in the (diagonal) transition matrix. The mode frequency $F_{\text {inst }}$ can be expressed as follows:

$$
\frac{F_{\text {inst }}}{F_{\text {syn }}}=m+m\left[\frac{\Delta F_{\mathrm{pwd}}+\Delta F_{\mathrm{dyn}}}{F_{\mathrm{syn}}}\right]
$$

where [8]

$$
\frac{\Delta F_{\mathrm{pwd}}+\Delta F_{\mathrm{dyn}}}{F_{\mathrm{syn}}}=-I_{b} \frac{G}{\sigma^{3}}\left[1-\frac{2}{\sqrt{\pi}} \frac{\Gamma(m+1 / 2)}{m !}\right] .
$$

Here $I_{b}$ is the average bunch current, $\sigma$ is the bunch length, and $G$ is a constant which, for our constant-voltage mode, is independent of $F_{\text {syn }}$ and energy. The first term in the square brackets in Eq. (8) corresponds to the PWD correction while the second term represents the dynamic correction. The latter decreases monotonically with increasing $m$ roughly as $1 / \sqrt{m}$. (A hypothetical decrease in the dynamic correction with increasing $m$ was noted earlier). The total correction vanishes identically for $m=1$, as required. Let us calibrate the constant $G$ against the experimental value $F_{\text {inst }} / F_{\text {syn }} \approx 7.5$ for $m=8$. Then from Eqs. (7)-(8) we calculate $F_{\text {inst }} / F_{\text {syn }} \approx 4.7,5.7,6.6$, and 7.5 for $m=5-8$, respectively, which are comparable to the $1.5 \mathrm{GeV}$ ratios in Fig. 6 and the upper ends of the $2.9 \mathrm{GeV}$ ratios in Fig. 8. Focusing solely on the $2.9 \mathrm{GeV}$ results, let us suppose that the dynamic correction $\Delta F_{\text {dyn }}$ is completely suppressed by damping at the initiation of each eigenmode step. Then we calculate $F_{\text {inst }} / F_{\text {syn }} \approx 4.5,5.4,6.3$, and 7.2 for $m=5-8$, respectively, which are comparable to the ratios at the beginning of the steps in Fig. 8. While encouraging, we emphasize that this discussion is merely intended to demonstrate that the slopes across the higher modes may be a consequence of radiation damping. The argument obviously cannot be extended down to $m=1$ without disturbing the necessary balance between the PWD and dynamic corrections.

\section{Instability thresholds}

As noted earlier, calculations of the instability thresholds have developed along two lines. We begin with the work of Bane et al. [9] which solves the Vlasov-Fokker-Planck (VFP) equation. This leads to a parametrization that 
depends on the two dimensionless parameters $\Pi$ and $S_{\mathrm{csr}}$ defined by

$$
\Pi=\frac{\sigma R^{1 / 2}}{(H / 2)^{3 / 2}} \quad S_{\mathrm{csr}}=\frac{I_{n} R^{1 / 3}}{\sigma^{4 / 3}}
$$

where $\sigma$ is the zero-current bunch length, $R$ is the orbital radius, and $H$ is the height of the vacuum chamber as defined by two parallel plates $(H=32 \mathrm{~mm})$. The normalized bunch current is defined by

$$
I_{n}=\frac{r_{0} N_{b}}{2 \pi \nu_{s o} \gamma(\Delta E / E)}
$$

where $N_{b}$ is the bunch population, $\nu_{s o}$ is the unmodified synchrotron tune, and $\Delta E / E$ is the rms relative energy spread. At threshold the quantities in Eq. (9) are related by the linear approximation

$$
\left(S_{\mathrm{csr}}\right)_{\mathrm{thr}} \approx 0.5+0.12 \text { П. }
$$

Given $\Pi$, we solve for $\left(S_{\text {csr }}\right)_{\text {thr }}$ and from Eqs. (9) and (10) deduce the theoretical threshold currents $I_{\text {theo }}$. The experimental thresholds $I_{b}^{\mathrm{thr}}$ were determined empirically as described earlier. Theory is compared with experiment at 1.5 and $2.9 \mathrm{GeV}$ in Fig. 9, where we plot the ratio $I_{b}^{\mathrm{thr}} / I_{\text {theo }}$ as a function of $\Pi$. The rapid rise below $\Pi \approx 1.5$ indicates a breakdown of the linear relationship described by Eq. (11)

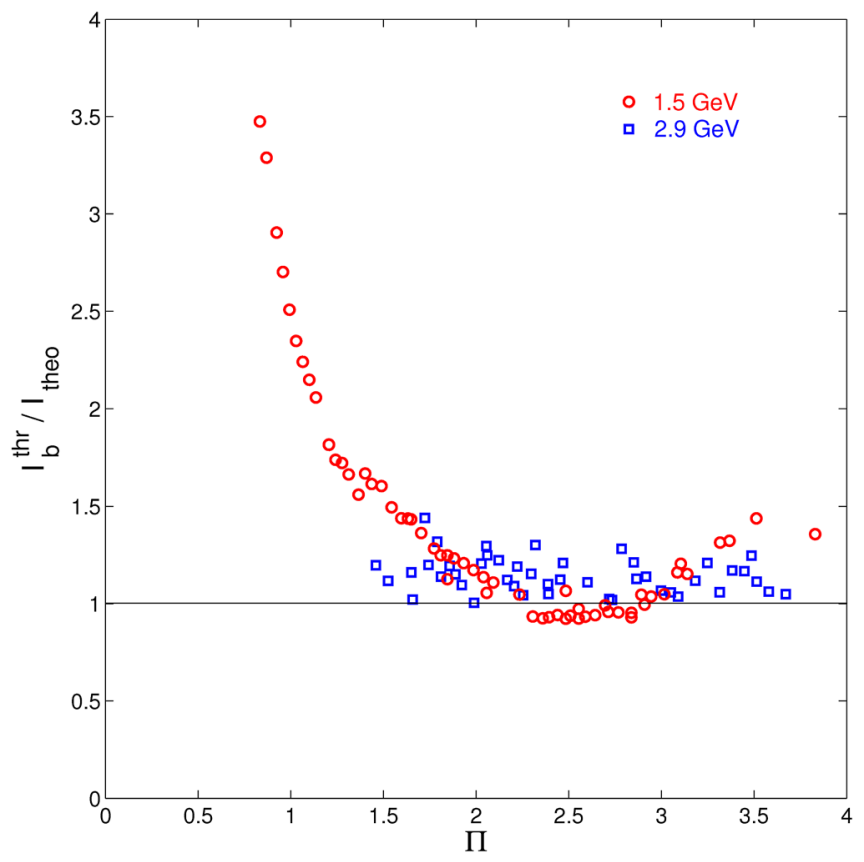

FIG. 9. Ratio of the experimental to theoretical threshold currents at 2.9 and $1.5 \mathrm{GeV}$ plotted against the shielding parameter $\Pi$ from the theory of Bane et al. [9]. The rapid rise below $\Pi \approx 1.5$ indicates a breakdown of the linear relationship Eq. (11).
(The results at 1.0,1.2, and $2.2 \mathrm{GeV}$ show a similar trend). The theory behind Eq. (11) assumes a so-called "strong" instability, one driven by azimuthal mode coupling [9]. However, we have suggested that the present measurements are more indicative of radial coupling, which fall under the category of so-called "weak" instabilities. We will continue this discussion of Fig. 9 following the introduction of Fig. 10 below.

The second approach to instability theory is based on linearization of the Vlasov equation, and here we focus on the recent study by Cai [10]. Near threshold, the bunch density is modeled as a static Haissinski distribution plus a small time-dependent perturbation which is expanded in terms of azimuthal modes. These are further decomposed into radial-like modes. This construct resembles Sacherer's original formalism [21] and presents a more intuitive approach to instability theory than portrayed by a direct attack on the VFP equation. Mode mixing, for example, becomes the driving force behind the onset of an instability. Bunch distortion is driven by the CSR wakefield as defined by the radiation impedance. In [10], the unshielded freespace radiation impedance is employed while Bane et al. [9] use the more realistic parallel-plate model.

From the formula for the free-space CSR wakefield and other considerations, Cai [10] argues that the instability

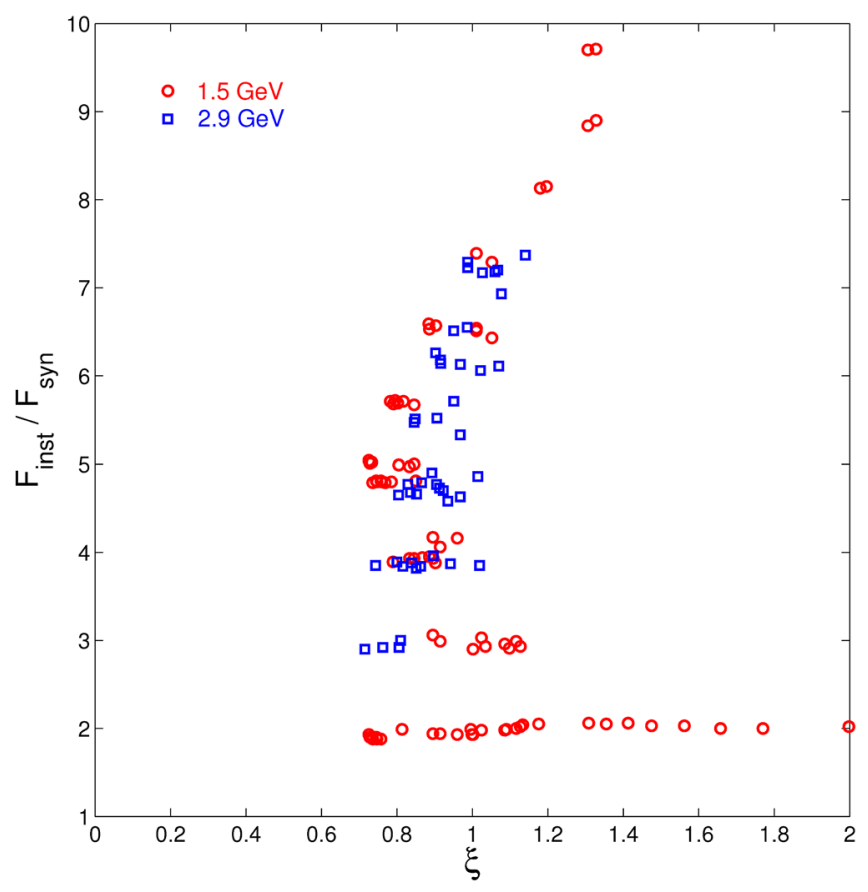

FIG. 10. Normalized instability frequencies at 2.9 and $1.5 \mathrm{GeV}$ as a function of the dimensionless parameter $\xi$ as defined by Cai [10] and Eq. (12) in the text. The data generally fall within the interval $\xi \approx 0.7-1.0$ in marked contrast to the threshold value $\xi_{\text {thr }}=0.50$ as predicted when shielding of the CSR impedance is ignored. Note the tendency to curve to the right with increasing mode number, equivalent here to increasing bunch length. 
theory should be characterized by the single dimensionless parameter $\xi$ defined by

$$
\xi=\frac{I_{n} R^{1 / 3}}{\sigma^{4 / 3}}
$$

where the normalized bunch current is given by Eq. (10) above. In Fig. 10 we plot $F_{\text {inst }} / F_{\text {syn }}$ as a function of $\xi$, and observe that most of the data fall within the interval $\xi \approx 0.7-1.0$. From a comparison of the instability thresholds for coasting beams versus bunched beams, Cai [24] deduced the relation $F=4 \pi \xi_{\text {thr }} / 3^{1 / 3}$ where $F=7.456$ is a theoretical constant and $\xi_{\text {thr }}$ is the threshold value of $\xi$. This gives $\xi_{\text {thr }}=0.856$, which is consistent with the results plotted in Fig. 10. We note that generally the bunch eigenmodes are simultaneously excited with respect to $\xi$, in agreement with theory, and furthermore the pattern in Fig. 10 bears a close resemblance to the corresponding pattern in [10], aside from the threshold at $\xi_{\text {thr }}=0.482$ for the unshielded impedance. That pattern is based on a radialcoupling model, further supporting our hypothesis that the present observations are driven by radial coupling. Note that $\xi$ is identical to the quantity $S_{\mathrm{csr}}$ as defined by Eq. (9). The free-space impedance corresponds to the limit $H \rightarrow \infty$ in the parallel-plate model, and with this limit in Eqs. (9) and (11), we would conclude that $\xi_{\text {thr }}=0.5$ in the unshielded limit.

We now return to a discussion of Fig. 9 and Eq. (11), noting again that $S_{\mathrm{csr}} \equiv \xi$. From Fig. 10 we see that experimental results are observed for values of $\xi$ between approximately 0.7 and 2.0. For values of $\Pi<1.5$, the theoretical predictions for $\xi$ given by Eq. (11) are lower than the observed values. Therefore, the theoretical threshold currents given by Eq. (12) and the observed $\xi$ will exceed the thresholds given by Eqs. (9)-(11) for $\Pi<1.5$. This leads to the calculated rise in Fig. 9. However, as $\Pi$ increases above 1.5 the predictions made using Eq. (11) become consistent with the observed values in Fig. 10 and therefore the ratios plotted in Fig. 9 approach unity. As a final comment, we note that the VFP calculations by Kuske [25] show thresholds higher than predicted by Eq. (11) for $\Pi<1$, while roughly following it for $\Pi>1$.

Given that all modes have a similar threshold in $\xi$, how then do we understand the sequential nature of the excitations as displayed in Figs. 5-8? Earlier, with respect to Fig. 5, we noted the approximate alignment of the line defined by Eqs. (2)-(4) with the start of each mode when plotted as a function of the zero-current bunch length. Let us pursue this and express Eq. (2) at the start of mode $m$ as

$$
\frac{F_{\text {inst }}}{F_{\text {syn }}}=m=\frac{2 \pi \sigma_{m}}{\lambda_{0}}
$$

where $\sigma_{m}$ is the zero-current bunch length at the initiation of the mode and $\lambda_{0}$ defined by Eq. (3) is the wavelength marking the peak of the CSR spectral distribution. [In writing Eq. (13) we are ignoring the frequency shifts due to PWD, etc.]. Treating the bunch length as the dependent variable, we have

$$
\sigma_{m}=m \frac{\lambda_{0}}{2 \pi}
$$

The relation between $\sigma_{m}$ and $\lambda_{0}$ is deeper than the simple argument that lead to Eq. (2) and thence to Eq. (14). We note that it is the real part of the radiation impedance that drives the coupling between radial modes [5]. It is also $\operatorname{Re} Z(\omega)$ that defines the instability growth rate. It follows that instability will first occur in that particular azimuthal mode whose power spectrum peaks near the maximum of $\operatorname{ReZ}(\omega)$, i.e., near $\lambda_{0}$ as we have defined it. Observation of CSR at a wavelength $\lambda_{0}$ means the power spectrum of the azimuthal mode $m$ must contain wavelengths comparable to $\lambda_{0}$. The power spectrum is generally expressed as a function of the dimensionless parameter $\sigma / \lambda$ where $\lambda$ is the spectral wavelength and $\sigma$ is the unperturbed bunch length [5]. The peak in the power spectrum is a strong function of the mathematical model describing the azimuthal mode of interest. We illustrate with two examples, classified according to their unperturbed bunch distributions, and give the bunch length that places the peak of the power spectrum at the wavelength $\lambda_{0}$. (i) Gaussian model: $\sigma_{m}=\sqrt{m} \frac{\lambda_{0}}{2 \pi}$ (ii) Parabolic model: $\sigma_{m} \approx m^{0.7} \frac{\lambda_{0}}{2 \pi}$. These are distinguished from each other, and from Eq. (14), solely by the exponential power of $m$ which is clearly model-dependent. Experiment seems to favor an exponential power closer to unity.

Let us now see how the relation Eq. (14) ties in with the bunch current, which is the usual threshold-defining parameter. Using Eq. (4), we note that the normalized bunch current as defined by Eq. (10) can be expressed in the form

$$
I_{n}=A \frac{I_{b}}{\sigma}
$$

where $A$ is a constant that depends on the cavity voltage, etc., but is independent of energy and energy spread, and $I_{b}$ is the average bunch current. Thus, the parameter $\xi$ in Eq. (12) takes the form

$$
\xi \propto \frac{I_{b}}{\sigma^{7 / 3}} .
$$

Denoting the threshold value of $\xi$ common to all modes by $\xi_{\text {thr }}$ (ignoring the dispersal in Fig. 10) we solve for the bunch current at the initiation of mode $m$ :

$$
I_{b}^{\mathrm{thr}}(m) \propto \xi_{\mathrm{thr}} \sigma_{m}^{7 / 3}
$$

where $\sigma_{m}$ is defined by Eq. (14). Moving now across the mode expressed as a function of $\sigma$, as in Fig. 5, we write in general 


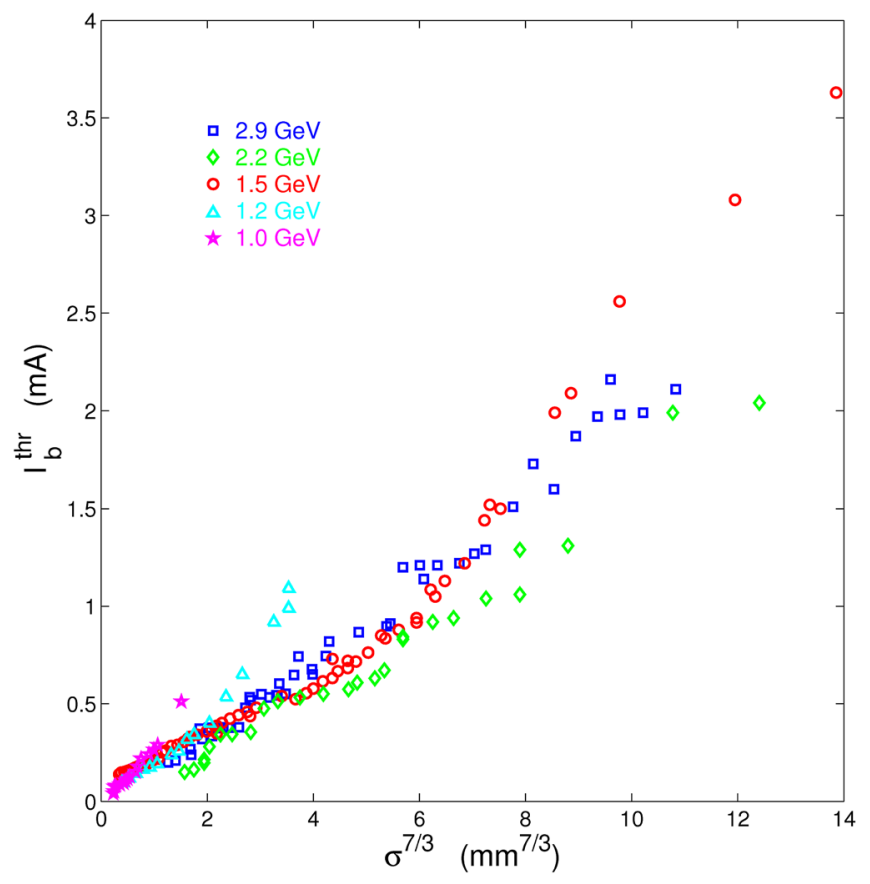

FIG. 11. Experimental threshold bunch current at all five energies plotted as a function of zero-current bunch length raised to the $7 / 3$ power. A linear description is expected if the threshold parameter $\xi_{\text {thr }}$ is a universal constant across all modes, which Fig. 10 shows is not a rigorous assumption. The synchrotron frequency, which is common to both parameters, varies from point to point.

$$
I_{b}^{\mathrm{thr}} \propto \xi_{\mathrm{thr}} \sigma^{7 / 3}
$$

where the current and bunch length share the same synchrotron frequency. We compare this relation with experiment in Fig. 11, where we show the threshold currents plotted as a function of $\sigma^{7 / 3}$ for all five energies. A linear dependence is expected if $\xi_{\text {thr }}$ is a common constant for all modes, and indeed the results are fairly linear out to a bunch length of $\sigma \approx 2 \mathrm{~mm}$. The subsequent upward slant can be traced to the curvature in $\xi$ with increasing $F_{\text {inst }}$ (i.e., with increasing $\sigma$ ) as evident in Fig. 10. In any event, Fig. 11 demonstrates an energy-independent correlation between the threshold current and the associated zero-current bunch length (at the same $F_{\text {syn }}$ ) over a wide range of the parameters.

\section{E. Coexisting modes}

The excitation functions displayed in Figs. 6-8 suggest the various eigenmodes are excited sequentially, one mode at a time. However, a closer examination shows that some modes coexist, meaning that two modes are simultaneously active in the same bunch. In other words, the phase-space expansion Eq. (1) contains both of these azimuthal modes, replacing the usual single-mode expansion. The coexistence of modes is evident in the time-domain spectra as two simultaneous oscillations in the CSR spectra at different frequencies. Generally, coexisting modes are confined to the transition boundaries between modes $m$ and $m+1$. This is plausible, since at the boundary the devolution of one mode is concurrent with the evolution of the subsequent mode, with both coexisting over a small interval in $F_{\text {syn }}$.

An interesting exception to the above rule is found in the $1.5 \mathrm{GeV}$ data in Fig. 6 associated with the $m=2$ mode. Coexisting $m=2$ and 3 modes are observed at $F_{\text {syn }}=7.2 \mathrm{kHz}$, not surprising since this marks the boundary between the two steps. What is unusual are the two strings of $m=2$ data following this transition boundary, from $F_{\text {syn }}=7.9-10.4 \mathrm{kHz}$ and $F_{\text {syn }}=13.0-14.6 \mathrm{kHz}$. Much of the $m=2$ data in the first string is found to coexist with the $m=3$ data directly above, while all the $m=2$ data in the second string coexists with a subset of data in the $m=5$ step above. Finally, we note a gradual decrease in $F_{\text {inst }} / F_{\text {syn }}$ for the $m=2$ mode with increasing $F_{\text {syn }}$. Perhaps these are all symptoms of an increasing incursion by azimuthal-mode mixing into what we perceive to be transitions driven by radial-mode mixing.

\section{CONCLUDING REMARKS}

The present measurements display clear evidence of longitudinal bunch excitations up through the $m=8$ mode. The modes appear to be excited sequentially as a function of bunch length or synchrotron frequency, although there is evidence of coexisting modes at the intermodal boundaries. A given mode crosses a finite span of bunch lengths or synchrotron frequencies before the next mode is excited. A systematic downward shift in the mode frequencies below integral multiples of the synchrotron frequency can be explained by a balance between the reduction in the synchrotron frequency from potential-well distortion and the increase in frequency from the dynamic correction. Focusing on the $2.9 \mathrm{GeV}$ data, the frequency of a given mode displays an upward slope when plotted against synchrotron frequency or bunch length, and the slope angle increases with mode number. An explanation may lie within the dynamic correction to the synchrotron frequency, wherein the correction varies in magnitude across the mode due to the changing influence of radiation damping. We observe a strong correlation of the slopes with synchrotron frequency, as demonstrated by comparing the 2.9 and $1.5 \mathrm{GeV}$ data, and this is consistent with the damping conjecture.

The excitation of a given mode depends on a number of parameters, including the synchrotron frequency $F_{\text {syn }}$, the bunch current at threshold, the unperturbed bunch length, and the beam energy. We have examined the correlations between these parameters and observe the following. We find that the mode frequency $F_{\text {inst }}$, normalized by $F_{\text {syn }}$, appears to be a linear function of the bunch length calculated for the same synchrotron frequency. The threshold current, on the other hand, is a highly nonlinear 
function of the bunch length. Both correlations are independent of the beam energy.

As yet, no theoretical calculations are available to provide a deeper understanding of the observations reported here. For reasons not yet understood, the VFP solvers do not predict excitations above the quadrupole $(m=2)$ mode, at least for the particular geometries studied $[3,6]$, with the exception of [23]. On the other hand, the linear model [10] exhibits azimuthal modes up to $m=15$ for the SLC Damping Ring. This incongruity clearly demands further theoretical study, especially in the threshold region. Much of the physics behind the longitudinal excitations is based on the concept of mode mixing, especially between the radial modes, and this is introduced explicitly into the linear theory in the course of the theory development. Cai [10], for example, includes up to 20 azimuthal and 20 radial modes in his model. The VFP equation, on the other hand, makes no explicit reference to mode structure since the concept, or its equivalent, is presumably subsumed within the nonlinear theory. That might suggest that the existing VFP solvers are not sufficiently sensitive to this underlying aspect.

\section{ACKNOWLEDGMENTS}

Research was funded by the Canadian Foundation for Innovation, the Natural Sciences and Engineering Research Council of Canada, the National Research Council Canada, the Canadian Institutes of Health Research, the Government of Saskatchewan, Western Economic Diversification Canada, and the University of Saskatchewan.

[1] J. Haissinski, Il Nuovo Cimento B 18, 72 (1973).

[2] G. Stupakov and S. Heifets, Phys. Rev. Accel. Beams 5, 054402 (2002).

[3] M. Venturini and R. Warnock, Phys. Rev. Lett. 89, 224802 (2002).

[4] J. B. Murphy, ICFA Beam Dynamics Newsletter 35, 20 (2004).

[5] K. Y. Ng, Physics of Intensity Dependent Instabilities (World Scientific, Singapore, 2006).

[6] M. Venturini, R. Warnock, R. Ruth, and J. A. Ellison, Phys. Rev. Accel. Beams 8, 014202 (2005).

[7] E. Roussel, C. Evain, C. Szwaj, and S. Bielawski, Phys. Rev. Accel. Beams 17, 010701 (2014).
[8] A. W. Chao, Physics of Collective Beam Instabilities in High Energy Accelerators (John Wiley \& Sons, New York, 1993).

[9] K. L. F. Bane, Y. Cai, and G. Stupakov, Phys. Rev. Accel. Beams 13, 104402 (2010).

[10] Y. Cai, Phys. Rev. Accel. Beams 14, 061002 (2011).

[11] P. Kuske, in Proceedings of the 23rd Particle Accelerator Conference, Vancouver, Canada, 2009 (IEEE, Piscataway, NJ, 2009), p. 4682.

[12] M. Ries, J. Feikes, P. Schmid, G. Wüstefeld, and A. Hoehl, in Proceedings of the 3rd International Particle Accelerator Conference, New Orleans, LA, 2012 (IEEE, Piscataway, NJ, 2012), p. 3030.

[13] W. Shields, R. Bartolini, G. Boorman, P. Karataev, A. Lyapin, J. Puntree, and G. Rehm, in IX International Symposium on Radiation from Relativistic Electrons in Periodic Structures (IOP Publishing, Egham, 2012).

[14] V. Judin, A.-S. Muller, M. Schwarz, and M. Klein, in Proceedings of the 4th International Particle Accelerator Conference, IPAC-2013, Shanghai, China, 2013 (JACoW, Shanghai, China, 2013), p. 2516.

[15] Model DXP-15 detector and Model FLP-15 low pass filter, Technical Report (Millitech Inc., 29 Industrial Dr. East, Northhampton, MA 01060).

[16] B. E. Billinghurst, J. C. Bergstrom, C. Baribeau, T. Batten, L. Dallin, T. E. May, J. M. Vogt, W. A. Wurtz, R. Warnock, D. A. Bizzozero, and S. Kramer, Phys. Rev. Lett. 114, 204801 (2015).

[17] P. R. Griffiths and J. A. de Haseth, Fourier Transform Infrared Spectroscopy (Wiley-Interscience, New York, 1986).

[18] R. B. Blackman and J.W. Tukey, The Measurement of Power Spectra from the Point of View of Communications Engineering (Dover Publications, New York, 1959) p. 98.

[19] P. Kuske, Third low emittance ring workshop (to be published).

[20] R. L. Warnock, in Proceedings of the 1991 Particle Accelerator Conference, San Francisco, CA, 1991 (IEEE, New York, 1991), p. 1824.

[21] F. J. Sacherer, IEEE Trans. Nucl. Sci. 24, 1393 (1977).

[22] B. W. Zotter, CERN Report No. LEP/TH 89-74, 1989.

[23] P. Kuske, in Proceedings of the International Computational Accelerator Physics Conference, Rostock-Warnemunde, Germany, 2012 (JACoW, Geneva, 2013), p. 2014.

[24] Y. Cai, in Proceedings of the 2nd International Particle Accelerator Conference, San Sebastiáán, Spain (EPS-AG, Spain, 2011), p. 3774.

[25] P. Kuske, in Proceedings of the 4th International Particle Accelerator Conference, IPAC-2013, Shanghai, China, 2013 (JACoW, Shanghai, China, 2013), p. 2014. 